\title{
Tonsil Size and Mallampati Score as Clinical Predictive Factors for Obstructive Sleep Apnea Severity in Children
}

\author{
Maen Zreaqat ${ }^{1}$, Rozita Hassan ${ }^{2}$, Samsudin $\mathrm{AR}^{3}$, Yasser Stas ${ }^{4}$, Abdulfatah Hanoun $^{5}$
}

\begin{abstract}
Aim and objective: The aim of this study was to determine the clinical utility of body mass index (BMI), tonsil size, and Mallampati scoring in predicting both the presence of and severity of pediatric obstructive sleep apnea (OSA).

Materials and methods: This prospective cross-sectional study comprised 78 growing children in the age range of 11-14 years with polysomnography (PSG)-proven OSA and 86 non-OSA corresponding controls. BMI, tonsil size (Friedman grading scale), and Mallampati score were determined for both groups, and related differences were assessed with a $t$-test, while their independent association with OSA severity was tested with a regression analysis. Statistical significance was set at $p<0.05$.

Results: Male gender, BMI, tonsil size, and Mallampati score were significantly higher in the OSA group $(p<0.05)$. A significant correlation was recorded between the Mallampati score and OSA severity $(p<0.01)$, but not with BMI or tonsil size $(p>0.05)$. For every 1 -point increase in the Mallampati scale, the apnea-hypopnea index (AHI) increased by more than five events per hour in the bivariate analysis and by more than three events per hour in the multivariate analysis.

Conclusion: Male gender, increased BMI, high tonsil, and Mallampati scores were clinical indicators of the presence of OSA. However, only Mallampati scale had a significant association with OSA severity. Clinical diagnostic indicators should be established and encouraged especially in community-based studies.

Clinical significance: Clinical diagnostic indicators are very useful in examining and screening children who are at risk of developing OSA as PSG is expensive and unsuitable for universal use in the pediatric population.

Keywords: Mallampati score, Obstructive sleep apnea, Tonsil size.

The Journal of Contemporary Dental Practice (2021): 10.5005/jp-journals-10024-3148
\end{abstract}

\section{INTRODUCTION}

Sleep disturbances can compromise somatic growth and result in neurobehavioral morbidities in the pediatric population, including daytime sleepiness, hyperactivity, irritability, and lower cognitive and learning performance. ${ }^{1-4}$ If gone untreated, OSA may carry serious health consequences resulting in cardiovascular and other systemic diseases. ${ }^{5,6}$ Sleep breathing disorders have a wide range of symptoms, from harmless primary snoring to obstructive sleep apnea syndrome (OSAS), a condition related to snoring with nighttime repetitive airway patency disruptions, and can even lead to life-threatening complications in the pediatric population. Being unaware of OSA and its potential complications in children may result in delayed diagnosis and unnecessary morbidity.

Anatomical and functional abnormalities of the upper airway may act as a key factor in the increased upper airway resistance and development of pediatric OSA where a reciprocal interaction has been reported between the upper airways and the craniofacial complex. Enlargement of the adenoids, tonsils, and neighboring structures causes direct narrowing of upper airways particularly at the level of oropharynx and hypopharynx leading to reduced upper airway patency. ${ }^{7,8}$ In addition, increased fatty tissues particularly in the tongue and pharyngeal tissues will affect negatively the upper airway patency due to reduced normal muscular tone during respiration. ${ }^{9}$

Overnight polysomnography (PSG) is considered the definitive method for predicting and diagnosing pediatric OSA and other sleep disturbances worldwide. It is a noninvasive intervention and is able to record respiratory, cardiovascular, and neural functions during sleep. However, PSG is costly and remains prohibitively
1,2,4,5 Orthodontic Department, School of Dental Sciences, Universiti Sains Malaysia, Kota Bharu, Malaysia

${ }^{3}$ Oral and Maxillofacial Surgery Department, University of Sharjah, Sharjah, United Arab Emirates

Corresponding Author: Rozita Hassan, Orthodontic Department, School of Dental Sciences, Universiti Sains Malaysia, Kota Bharu, Malaysia, Phone: +0060199886161, e-mail: rozitakb@usm.my

How to cite this article: Zreaqat $M$, Hassan $R$, Samsudin $A R$, et al. Tonsil Size and Mallampati Score as Clinical Predictive Factors for Obstructive Sleep Apnea Severity in Children. J Contemp Dent Pract 2021;22(7):850-853.

Source of support: Nil

Conflict of interest: None

expensive for community-based studies. Tonsil size (adenotonsillar hypertrophy) and Mallampati score (oropharyngeal crowding) are based on the visualization of the oropharynx. They are simple, noninvasive, and inexpensive diagnostic approaches that require no special equipment. The tonsil size evaluation (known also as Friedman scale) indicates the free pharyngeal airway space that has a crucial role in upper airway collapsibility during sleep. Tonsil size scoring was shown to be reproducible and consistent in the pediatric population making it suitable for clinical and research environments. ${ }^{10}$ On the contrary, the Mallampati score comprises a visual assessment of the distance from the tongue base to the roof of the mouth. The soring requires cooperative children who allow visualization of their oropharynx. Mallampati score proved

(0) Jaypee Brothers Medical Publishers. 2021 Open Access This article is distributed under the terms of the Creative Commons Attribution 4.0 International License (https://creativecommons.org/licenses/by-nc/4.0/), which permits unrestricted use, distribution, and non-commercial reproduction in any medium, provided you give appropriate credit to the original author(s) and the source, provide a link to the Creative Commons license, and indicate if changes were made. The Creative Commons Public Domain Dedication waiver (http://creativecommons.org/publicdomain/zero/1.0/) applies to the data made available in this article, unless otherwise stated. 
to have a high sensitivity score (89.7\%) in OSA prediction ${ }^{11}$ with a satisfactory level of reliability when classifying children with mild or severe symptoms. ${ }^{12}$ The American Academy of Sleep Medicine (AASM) has proposed tonsil size and Mallampati score as potential anatomic risk factors in OSA pathogenesis and may serve as independent predictors of pediatric OSA. ${ }^{13}$ However, the evidence of their association with the severity of pediatric OSA is still lacking. The aim of this study was to determine the clinical utility of BMI, tonsil size, and Mallampati scoring in the objective assessment of pediatric OSA severity.

\section{Materials and Methods}

This prospective study was approved by the Institutional Review Board of the Universiti Sains Malaysia. Informed consent forms were obtained from participants and/or their parents prior to assessment while they received explanatory letters discussing the study clearly. The sample study comprised 78 growing children in the age range of 11-14 years with a PSG-proven OSA ( $\mathrm{AHI}>5.0$ /hour) and 86 non-OSA corresponding controls who were at low-risk OSA as assessed by the Pediatric Sleep Questionnaire (PSQ). Those with chronic pulmonary diseases, craniofacial malformations, and a history of tonsillectomy were excluded from the study. Patients with complete relevant PSG data underwent clinical evaluation of demographic measures, tonsil size, and Mallampati scoring. All assessments were performed by the same investigator (M.Z.).

Patients were diagnosed with OSA after which they underwent a standard overnight PSG by sleep medicine specialists at a pediatric sleep lab and referred later to orthodontic clinic. The PSG (model Alice 4; Respironics Inc., Murrysville, PA, USA) was performed following standards set by the AASM. The following parameters were monitored and recorded (Table 1): Electroencephalography (F4/A1, C4/ A1, O2/A1), electrooculography (bilateral), electrocardiography, electromyography, oronasal airflow (assessed with an oronasal thermistor), and chest and abdominal wall respiratory movements (assessed with Piezo sensors). Apnea was defined as the cessation of breath (airflow) lasting for at least two respiratory cycles (breaths) with the presence of respiratory effort throughout the entire period of absent airflow where hypopnea was defined as a drop in peak airflow that is $\geq 30 \%$ of pre-event baseline, for the duration

Table 1: PSG data of OSA group

\begin{tabular}{lc}
\hline PSG variable & Mean (SD) \\
\hline Total sleep time (hour) & $7.9 \pm 0.9$ \\
Sleep efficiency (\%) & $88.1 \pm 0.3$ \\
Sleep stage (\%) & \\
1 & $5.6 \pm 2.4$ \\
2 & $42.4 \pm 12.7$ \\
SWS & $37.6 \pm 14.1$ \\
REM sleep & $18.3 \pm 4.7$ \\
Apnea index (events/hour) & $5.9 \pm 4.3$ \\
AHI (events/hour) & $13.5 \pm 6.9$ \\
Oxygen desaturation index (events/hour) & $12.5 \pm 8.2$ \\
Lowest SpO & (\%) \\
Average $\mathrm{SpO}_{2}$ (\%) & $88.3 \pm 6.4$ \\
Arousal index (events/hour) & $96.5 \pm 2.2$ \\
\hline
\end{tabular}

SWS, slow-wave sleep; $\mathrm{REM}$, rapideyemovement; $\mathrm{SpO}_{2}$, oxygen desaturation as measured by pulse oximetry of at least two breaths in association with either $\geq 3 \%$ of oxygen desaturation or an arousal. Apnea-hypopnea index (AHI), being the most important indicator of OSA severity, was defined as the number of apneas and hypopneas per hour of total sleep time. On the contrary, the use of a highly validated screening questionnaire (PSQ) helped in excluding sleep disorders within controls.

Tonsil size was evaluated in the sitting position using the Friedman grading scale that comprises 5 grades as follows: Grade 0 , tonsils fit within tonsillar fossa; grade 1, tonsils occupy $<25 \%$ of space between pillars (oropharyngeal width); grade 2, tonsils occupy $<50 \%$ of space between pillars; grade 3 , tonsils occupy $<75 \%$ of space between pillars; and grade 4 , tonsils occupy $>75 \%$ of space between pillars. The score was assessed by asking the patient, in a sitting posture, to open his or her mouth and to protrude the tongue as much as possible. The Mallampati score was obtained in the sitting position as well. Being awake and cooperative, patients were asked to open their mouth and stick out their tongue and assessed based upon the pharyngeal structures that are visible. The scale comprises four categories as follows: Class I, soft palate, uvula, fauces, and pillars visible; class II, soft palate, major part of uvula, and fauces visible; class III, soft palate, and the base of uvula visible; and class IV, only hard palate visible. In addition, body mass index [BMI $\left(\mathrm{kg} / \mathrm{m}^{2}\right)$ ] was calculated for both groups based on specific growth charts described by Centers for Disease Control as follows: underweight, BMI below 18.5; normal, between 8.5 and 24.9; overweight, between 25 and 29.9; and obese, 30 and above.

\section{Statistical Analysis}

Differences between OSA and non-OSA groups in terms of BMI, tonsil size and, Mallampati score were assessed with a $t$-test with a statistical significance set at $p$-value $<0.05$. The independent association between BMI, tonsil size, Mallampati score, and OSA severity was determined with a multiple linear regression analysis on a continuous scale. The regression model was checked with Hosmer-Lemeshow test and showed to have good fitness ( $p=0.56$ ).

\section{Results}

Table 2 shows the features of both groups in terms of age, sex, BMI, tonsil size, Mallampati score, and related differences. The mean ages of OSA and control groups were 10.8 and 11.4 years, respectively $(p=0.241)$. The sex distributions showed that males were predominant in the OSA group (49/29) compared to the controls $(46 / 40)$ with $p=0.017$. BMI was significantly higher $(p=0.031)$ in the OSA group (26.9) compared to controls (20.8). Tonsil size was significantly higher in the OSA group $(p=0.007)$. Tonsil size was distributed from grades 0 to IV in the OSA group with a mean of 2.56 and from grades 0 to III in the control group with a mean of 1.92. On the contrary, the Mallampati score was significantly higher $(p<0.001)$ in the OSA group (mean, 2.64) compared to controls (mean, 1.41). A significant correlation was recorded between the Mallampati score and OSAS severity, but not with BMI or tonsil size (Table 3). The regression model (Table 4) showed that for every 1-point increase in the Mallampati scale, the AHI increased by more than five events per hour in the bivariate analysis and by more than three events per hour in the multivariate analysis $(p<0.05)$.

\section{Discussion}

Physical examination and evaluation of clinical indicators prior to PSG are useful diagnostic measures for identifying sleep 
disturbances. There is a general consensus that male gender, increased BMI, high tonsil grade, and high Mallampati score are associated with an increased risk of OSA. However, the evidence about the association of these factors with the severity of OSA is conflicting. Males had higher OSA prevalence when compared to females ( $p=0.0 .017)$, which is in an agreement with a majority of OSA epidemiology-based studies. ${ }^{14}$ There is an agreement that the male gender acts as a risk factor in sleep disturbances although the etiology responsible for this predilection is still unclear. Some reports have proposed that hormonal differences and variation in body fat distribution among both genders may have a role..$^{15,16}$

Increased BMI is another risk factor of developing OSA. The bidirectional relationship between increased BMI and OSA has been highlighted by the mainstream sleep research. The adipose tissue in the tongue and neck area is suggested to reduce the normal tone of respiratory muscles experienced during respiration and alter the functional residual capacity and other lung mechanics that

Table 2: Clinical findings of OSA group and controls

\begin{tabular}{lllr}
\hline & \multicolumn{1}{c}{ Non-OSA } & OSA & \\
& $N=86$ & $N=78$ & p value \\
\hline Age & 11.4 & 10.8 & 0.241 \\
Sex-male/female & $46 / 40$ & $49 / 29$ & 0.017 \\
BMI & $20.8(3.6)$ & $26.9(4.1)$ & 0.031 \\
Tonsil size & & & 0.007 \\
TS 0 & 19 & 4 & \\
TS 1 & 56 & 41 & \\
TS 2 & 10 & 21 & \\
TS 3 & 1 & 9 & \\
TS 4 & 0 & 3 & \\
Mallampati score & & & \\
MS 1 & 58 & 7 & \\
MS 2 & 22 & 23 & \\
MS 3 & 5 & 39 & \\
MS 4 & 1 & 9 & \\
\hline
\end{tabular}

Significant at $p<0.05$

Table 3: Apnea-Hypopnea index (AHI) for tonsil size and Mallampati scores-OSA group

\begin{tabular}{lcr}
\hline No. & AHI (events/hour) & (SD) \\
\hline Tonsil size $(n=78)$ & & \\
1 & $4(5.1 \%)$ & $1.88(2.91)$ \\
2 & $41(52.7)$ & $2.54(3.26)$ \\
3 & $21(26.9 \%)$ & $4.60(3.04)$ \\
4 & $9(11.5 \%)$ & $6.56(3.55)$ \\
Mallampati scores $(n=78)$ & & \\
1 & $7(9.0 \%)$ & $2.41(4.60)$ \\
2 & $23(29.5 \%)$ & $7.74(3.97)$ \\
3 & $39(50.0)$ & $12.44(4.53)$ \\
4 & $9(11.5 \%)$ & $17.63(4.59)$ \\
\hline
\end{tabular}

may lead to mechanical restriction of airflow. ${ }^{17,18}$ However, these mechanisms do not explain why nonobese and lean individuals may develop OSA. Although increased BMI indicated the presence of OSA, our logistic regression model did not demonstrate positive correlation between $\mathrm{BMI}$ and $\mathrm{AHI}$ (OSA). This mirrors several previous publications where overweight or obesity does not correlate with OSA severity. ${ }^{19-21}$ On the contrary, Friedman et al. found that increased BMI correlated significantly with OSA severity. ${ }^{22}$ In a multiethnic study, Sutherland et al. identified clinical anatomic risk factors in sleep disturbances and found that weight gain is an important contributor to AHI severity, particularly in South American populations. ${ }^{23}$

Tonsils appear around the 4th or 5th months of gestation and continue to grow between the 6th and 8th years of life and begin atrophy shortly after that. However, in children with OSA, enlargement is extended until 8-14 years old. ${ }^{24}$ Increased tonsil size (tonsillar hypertrophy) is an independent risk factor for OSA as shown by the significant increase of tonsil size scoring in OSA compared to controls, which is a common finding in previous reports. However, the association between tonsil size and OSA severity was poor $(p=0.13)$. The literature has a lot of conflicting evidence regarding the role of using tonsil size grading in the assessment of OSA severity. Tagaya et al. and Øverland et al. reported a mild association in 2- to 6-year-old children (preschool children) ${ }^{25,26}$ while Friedman et al. found a significant correlation $(p<0.001)$ in a 172 OSA patients. ${ }^{22}$ On the contrary, Nolan and Brietzke found that the association between subjective pediatric tonsil size and objective OSA severity was weak at best. ${ }^{27}$ Similarly, Tang et al. concluded that tonsil size did not correlate with OSA severity. ${ }^{28}$ It seems that this association becomes stronger with younger age due to the relatively larger size of the tonsils.

OSA group had higher Mallampati scores compared to non-OSA controls, which is a consistent finding among most publications. In addition, our results showed a strong association between the Mallampati scale and OSA severity. Several studies have shown the ability of the Mallampati score to predict OSA severity. Nuckton et al. and Kumar et al. found an independent association between Mallampati score and both presence and severity of OSA. ${ }^{29,10}$ Su et al. found that Mallampati scores were associated with OSA severity in both preschool and school children. ${ }^{30} \mathrm{Kljajic}$ et al. found that the Mallampati score has the most significant correlation with $\mathrm{AHI}(p<0.001)$ among all clinical indicators. ${ }^{31}$ It seems that Mallampati scores can reflect the nature in upper airway patency where multifactorial interactions, such as neuromuscular response and anatomic features, may determine airway collapsibility during sleep. Only two studies reported that the association between Mallampati score and AHI was insignificant. ${ }^{23,26}$ However, it should be noted that scoring may be affected by cooperation from the child and the experience of the physician assigning the scoring.

Table 4: Risk factors association with AHI (OSA severity)

\begin{tabular}{lcccccc}
\hline & \multicolumn{2}{c}{ Bivariate } & & \multicolumn{2}{c}{ Multivariate } \\
\cline { 2 - 3 } \cline { 6 - 7 } Variable & Coefficient $(95 \% \mathrm{Cl})$ & $p$ value & & Coefficient $(95 \% \mathrm{Cl})$ & $p$-value \\
\hline BMI & $1.3(0.7,2.3)$ & 0.21 & & $0.8(0.4,1.9)$ & 0.34 \\
Tonsil size & $3.1(2.5,6.4)$ & 0.21 & & $5.3(1.7,8.0)$ & 0.30 \\
Mallampati score & $5.3(2.2,8.0)$ & $<0.01$ & & $3.2(0.7,11.7)$ & 0.04 \\
\hline
\end{tabular}

Significance $(p<0.05)$ 
One limitation in this study is that tonsil size and Mallampati scoring have been assessed in the supine position, while OSA usually occurs during sleep. Camacho and colleagues found that the minimum cross-sectional area of upper airways decreased significantly when the patient was scanned with cone-beam computed tomography in supine compared to upright position due to a retrodisplacement of the base of the tongue and epiglottis in the supine position. ${ }^{32}$ In general, the results of this study support previous assumptions that reduced upper airway patency due to anatomical narrowing of the airway passage is responsible for respiratory disorders during sleep and may be correlated positively with OSA severity in children. However, a reliable diagnosis of OSA based on clinical examination alone remains uncertain.

\section{Conclusion}

Male gender, increased BMI, high tonsil, and Mallampati scores were clinical indicators of the presence of OSA. However, only Mallampati scale was associated with the severity of OSA. Due to the financial burden of PSG, clinical diagnostic indicators should be established and encouraged especially in community-based studies.

\section{References}

1. Ohida T, Osaki Y, Doi Y, et al. An epidemiologic study of self-reported sleep problems among Japanese adolescents. Sleep 2004;27(5): 978-985. DOI: 10.1093/sleep/27.5.978.

2. Alfano CA, Zakem AH, Costa NM, et al. Sleep problems and their relation to cognitive factors, anxiety, and depressive symptoms in children and adolescents. Depress Anxiety 2009;26(6):503-512. DOI: 10.1002/da.20443.

3. Taras H, Potts-Datema W. Sleep and student performance at school. J Sch Health 2005;75(7):248-254. DOI: 10.1111/j.1746-1561.2005.00033.x.

4. Ayas NT, Owens RL, Kheirandish-Gozal L. Update in sleep medicine 2014. Am J Respir Crit Care Med 2015;192(4):415-420. DOI: 10.1164/ rccm.201503-0647UP.

5. Jensen ME, Gibson PG, Collins CE, et al. Airway and systemic inflammation in obese children with asthma. Eur Respir J 2013;42(4):1012-1019. DOI: 10.1183/09031936.00124912.

6. Spruyt K, Molfese DL, Gozal D. Sleep duration, sleep regularity, body weight, and metabolic homeostasis in school-aged children. Pediatrics 2011;127(2):345-352. DOI: 10.1542/peds.2010-0497.

7. Liistro G, Rombaux P, Belge C, et al. High Mallampati score and nasal obstruction are associated risk factors for obstructive sleep apnoea. Eur Respir J 2003;21(2):248-252. DOI: 10.1183/09031936.03.00292403.

8. Ikävalko T, Närhi M, Eloranta AM, et al. Predictors of sleep disordered breathing in children: the PANIC study. Eur J Orthod 2018;40(3): 268-272. DOI: 10.1093/ejo/cjx056.

9. Certal V, Catumbela E, Winck JC, et al. Clinical assessment of pediatric obstructive sleep apnea: a systematic review and meta-analysis. Laryngoscope 2012;122(9):2105-2114. DOI: 10.1002/lary.23465.

10. Kumar DS, Valenzuela D, Kozak FK, et al. The reliability of clinical tonsil size grading in children. JAMA Otolaryngol Head Neck Surg 2014;140(11):1034-1037. DOI: 10.1001/jamaoto.2014.2338.

11. Kljajić Z, Roje Ž, Bečić K, et al. Formula for the prediction of apnea/ hypopnea index in children with obstructive sleep apnea without polysomnography according to the clinical parameters: is it reliable? Int J Pediatr Otorhinolaryngol 2017;100:168-173. DOI: 10.1016/j. ijporl.2017.06.032.

12. Kljajic Z, Glumac S, Deutsch JA, et al. Feasibility study of determining a risk assessment model for obstructive sleep apnea in children based on local findings and clinical indicators. Int J Pediatr Otorhinolaryngol 2020;135(6):110081. DOI: 10.1016/j.ijporl.2020.110081.

13. Berry RB, Brooks R, Gamaldo C, et al. AASM Scoring Manual Updates for 2017 (Version 2.4). J Clin Sleep Med 2017;15(5):665-666. DOI: 10.5664/jcsm.6576.
14. Gill Al, Schaughency E, Galland BC. Prevalence and factors associated with snoring in 3-year-olds: early links with behavioral adjustment. Sleep Med 2012;13(9):1191-1197. DOI: 10.1016/j.sleep.2012.05.007.

15. Fietze I, Laharnar N, Obst A, et al. Prevalence and association analysis of obstructive sleep apnea with gender and age differencesResults of SHIP-Trend. J Sleep Res 2019;28(5):e12770. DOI: 10.1111/ jsr.12770.

16. Barewal RM. Obstructive sleep apnea: the role of gender in prevalence, symptoms, and treatment success. Dent Clin North Am 2019;63(2):297-308. DOI: 10.1016/j.cden.2018.11.009.

17. Hamilton GS, Joosten SA. Obstructive sleep apnoea and obesity. Aust Fam Physician 2017;46(7):460-463. DOI: 10.1016/j. smrv.2012.08.002.

18. Tuomilehto H, Seppä J, Uusitupa M. Obesity and obstructive sleep apnea-clinical significance of weight loss. Sleep Med Rev 2013;17(5):321-329. DOI: 10.1016/j.smrv.2012.08.002.

19. Isaiah A, Spanier AJ, Grattan LM, et al. Predictors of behavioral changes after adenotonsillectomy in pediatric obstructive sleep apnea: a secondary analysis of a randomized clinical trial. JAMA Otolaryngol Head Neck Surg 2020;146(10):1-9. DOI: 10.1001/ jamaoto.2020.2432.

20. Kohler M, Lushington K, Couper R, et al. Obesity and risk of sleep related upper airway obstruction in Caucasian children. J Clin Sleep Med 2008;4(2):129-136. PMC2335392/18468311.

21. Redline S, Amin R, Beebe D, et al. The childhood adenotonsillectomy trial (CHAT): rationale, design, and challenges of a randomized controlled trial evaluating a standard surgical procedure in a pediatric population. Sleep 2011;34(11):1509-1517. DOI: 10.5665/ sleep.1388.

22. Friedman $M$, Tanyeri $H$, La Rosa $M$, et al. Clinical predictors of obstructive sleep apnea. Laryngoscope 1999;109(12):1901-1907. DOI: 10.1097/00005537-199912000-00002.

23. Sutherland K, Keenan BT, Bittencourt L, et al. A global comparison of anatomic risk factors and their relationship to obstructive sleep apnea severity in clinical samples. J Clin Sleep Med 2019;15(4):629-639. DOI: $10.5664 / \mathrm{jcsm} .7730$.

24. Kaditis AG, Lianou L, Hatzinikolaou S, et al. Tonsillar size in 2- to 14-year-old children with and without snoring. Pediatr Pulmonol 2009;44(12):1216-1222. DOI: 10.1002/ppul.21126.

25. Tagaya M, Nakata S, Yasuma F, et al. Relationship between adenoid size and severity of obstructive sleep apnea in preschool children. Int J Pediatr Otorhinolaryngol 2012;76(12):1827-1830. DOI: 10.1016/j. ijporl.2012.09.010.

26. Øverland B, Berdal H, Akre H. Obstructive sleep apnea in 2-6 year old children referred for adenotonsillectomy. Eur Arch Otorhinolaryngol 2019;276(7):2097-2104. DOI: 10.1007/s00405-019-05362-3.

27. Nolan J, Brietzke SE. Systematic review of pediatric tonsil size and polysomnogram-measured obstructive sleep apnea severity. Otolaryngol Head Neck Surg 2011;144(6):844-850. DOI: 10.1177/0194599811400683.

28. Tang A, Benke JR, Cohen AP, et al. Influence of tonsillar size on OSA improvement in children undergoing adenotonsillectomy. Otolaryngol Head Neck Surg 2015;153(2):281-285. DOI: 10.1177/0194599815583459.

29. Nuckton TJ, Glidden DV, Browner WS, et al. Physical examination: Mallampati score as an independent predictor of obstructive sleep apnea. Sleep 2006;29(7):903-908. DOI: 10.1093/sleep/29.7.903.

30. Su MS, Zhang HL, Cai XH, et al. Obesity in children with different risk factors for obstructive sleep apnea: a community-based study. Eur J Pediatr 2016;175(2):211-220. DOI: 10.1007/s00431-015-2613-6.

31. Kljajic Z, Glumac S, Deutsch JA, et al. Feasibility study of determining a risk assessment model for obstructive sleep apnea in children based on local findings and clinical indicators. Int J Pediatr Otorhinolaryngol 2020;135:110081. DOI: 10.1016/j.ijporl.2020.110081.

32. Camacho M, Capasso R, Schendel S. Airway changes in obstructive sleep apnoea patients associated with a supine versus an upright position examined using cone beam computed tomography. J Laryngol Otol 2014;128(9):824-830. DOI: 10.1017/S0022215114001686. 